\title{
TEM Study on PbS Quantum Dots made by Atomic Layer Deposition and Their Behavior under E-beam Irradiation
}

\author{
Hee Joon Jung*, Neil P. Dasgupta**, Fritz B. Prinz***, and Robert Sinclair* \\ *Department of Materials Science \& Engineering, Stanford University, Stanford, CA, USA \\ **Department of Mechanical Engineering, Stanford University, Stanford, CA, USA
}

Lead Sulfide $(\mathrm{PbS})$ which has a low bandgap $(0.41 \mathrm{eV})$ is a strong candidate for a quantum confining medium in the photovoltaics field. Our previous study demonstrated a thickness-dependence of the bandgap of $\mathrm{PbS}$ thin films fabricated by atomic layer deposition (ALD); the bandgap of $\mathrm{PbS}$ can be tuned up to almost $2.0 \mathrm{eV}$, significantly beyond its bulk bandgap $(0.41 \mathrm{eV})$ [1]. It is of great interest to explore an even lower dimensional structure such as quantum dots (QDs) for bandgap tuning, and this requires high resolution electron microscopy analysis.

In this study, we performed a structural study of PbS QDs fabricated by ALD. We focused on nucleation and island growth of $\mathrm{PbS}$ on different substrate materials during the first few tens of ALD cycles. We deposited PbS QDs onto TEM grids with a 40nm thick amorphous $\mathrm{SiO}_{2}$ support film. Also, QDs were deposited onto an ALD-grown $\mathrm{Al}_{2} \mathrm{O}_{3}$ film on the same $\mathrm{SiO}_{2} \mathrm{TEM}$ grids. Crystalline $\mathrm{PbS}$ QDs formed in both conditions. The density and size of the QDs increased with the number of ALD cycles, with occasional bridging between islands, up to at least 20 cycles as shown in Fig. 1. Interestingly, nucleation and growth behavior is dependent on substrate material as shown in Fig. 1-a and 1-b. This demonstrates an ability to create PbS QD structures with tunable density and size by selection of substrate materials and ALD cycles.

After exposing ALD-grown PbS QDs to a $200 \mathrm{keV}$ TEM beam under high vacuum, we observed enhanced coalescence of QDs after the beam exposure which increased the spacing between QDs. This creates well-isolated and circle-shaped QDs as can be seen from Fig. 2-a-1 and 2-b-1. While contrast of an as-deposited QD is even over the whole particle, the contrast at the center of the same QD is darker than that of outside after e-beam exposure as shown in Fig. 2-a-2 and 2-b-2. This is presumably explained by the thickness of the QD center after irradiation being thicker than periphery. Fig. 3 represents snap-shots of an in-situ TEM video taken during 6 min -9 min of the beam exposure. We observed that larger dots grow at the expense of smaller dots, which is consistent with an Ostwald ripening process. At the same time, the size of QDs decreased with increasing exposure time as can be seen in Fig.3.

In conclusion, TEM study of PbS QDs on different surface materials by ALD was performed and showed different behavior of nucleation and growth. And isolated circle-shape QDs with simultaneous coalescence and shrinkage of QDs were observed by in-situ TEM during e-beam irradiation.

[1] N. P. Dasgupta, W. Lee and F. B. Prinz, Chem. Mater. 21, 3973 (2009).

[2] This research is supported in part of the Center on Nanostructuring for Efficient Energy Conversion (CNEEC), an Energy Frontier Research Center funded by the U.S. Department of Energy, Office of Science, Office of Basic Energy Sciences under Award Number (DE-SC0001060). 

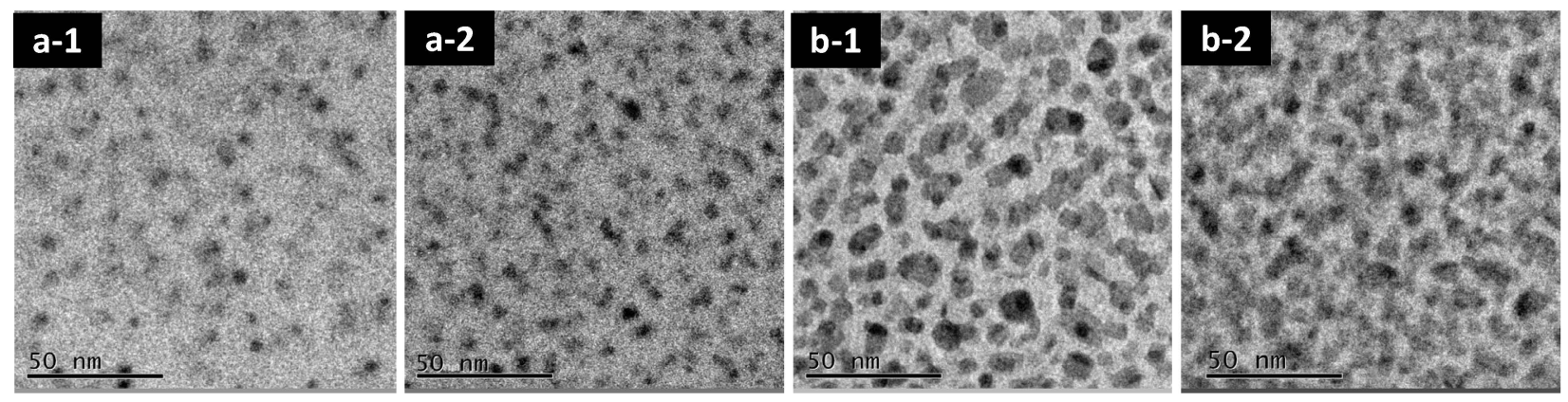

FIG. 1. BFTEM images of ALD PbS QDs on $\mathrm{SiO}_{2}$ and $\mathrm{Al}_{2} \mathrm{O}_{3}$ surface with different ALD cycles: (a-1) 10 cycles ALD on $\mathrm{SiO}_{2}$, (a-2) 20cycles ALD on $\mathrm{SiO}_{2}$, (b-1) 10 cycles ALD on $\mathrm{Al}_{2} \mathrm{O}_{3}$, (b-2) 20 cycles ALD on $\mathrm{Al}_{2} \mathrm{O}_{3}$.

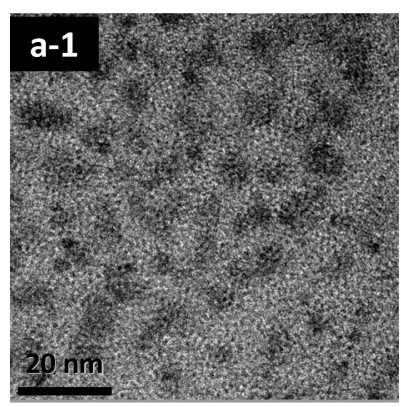

(a-1) As-deposited (Low mag.)

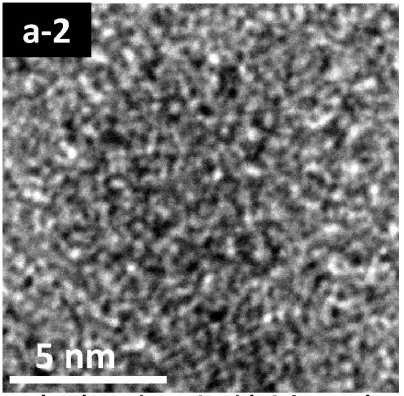

(a-2) As-deposited (High mag.)

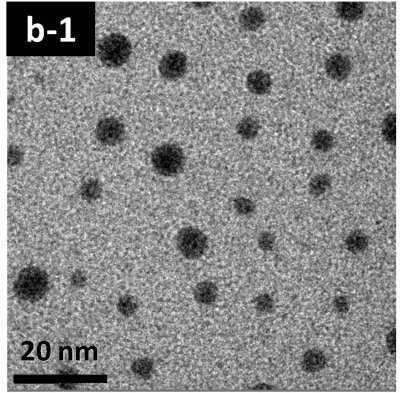

(b-1) After 11 min (Low mag.)

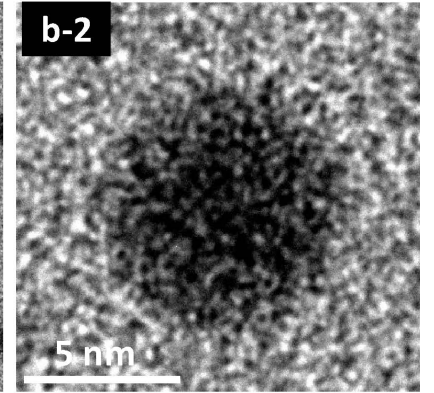

(b-2) After 11 min (High mag.)

FIG. 2. BFTEM images of $\mathrm{PbS}$ QDs on $\mathrm{SiO}_{2}$ surface by atomic layer deposition for (a) as-deposited and (b) 11 min electron beam exposure. Isolated $\mathrm{PbS}$ QDs with circular shape formed after e-beam irradiation.

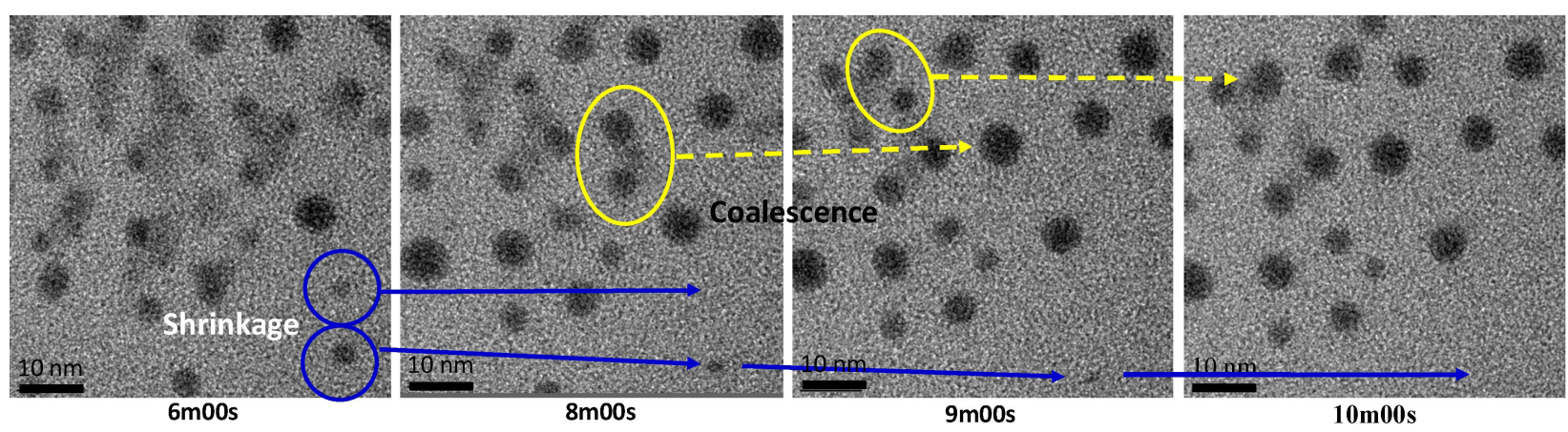

FIG. 3. In-situ TEM snap shots of PbS QDs during e-beam irradiation: Sublimation and coalescence are observed simultaneously during irradiation. 